\title{
Effect of Nitrogen Levels and Picking of Green Fruits on the Fruit and Seed Production of Okra (Abelmoschus Esculentus (L.) Moench)
}

\author{
M. MoniRUZZAMAN ${ }^{*}$ \& A. K. M. QUAMrUZZaman ${ }^{2}$ \\ ${ }^{1}$ ARS, Raikhali, Bangladesh Agricultural Research Institute, Chandraghona, Rangamati Hill District, \\ Bangladesh \\ ${ }^{2}$ Vegetable Section, HRC, Bangladesh Agricultural Research Institute, Joydebpur, Gazipur, \\ Bangladesh
}

\begin{abstract}
A field experiment on okra taking the variety BARI Dharos-1 with four nitrogen levels (0, 75, 125 and $175 \mathrm{~kg} / \mathrm{ha}$ ) and six levels of green fruit picking (no green fruit picking, first two, first four, first six, first eight and first ten green fruit picking) was conducted at the Agricultural Research Station, Raikhali, Rangamati Hill District to find out the optimum $N$ level and suitable number of green fruit picking during the summer seasons of 2005 and 2006 for the highest economic benefit. The plants were grown for both vegetable and seed production. Growth parameters, green fruit as well as dry fruit characters, seed yield attributes were significantly affected by different levels of $N$ and green fruit picking. Nitrogen levels and green fruit picking increased number of dry fruits per plant, number of seeds per fruit, weight of seeds per fruit and seed yield. Application of $N @ 125 \mathrm{~kg} / \mathrm{ha}$ gave the highest fruit and seed yield of okra followed by $175 \mathrm{~kg} \mathrm{~N} / \mathrm{ha}$. These two $\mathrm{N}$ levels also produced maximum 100-seed weight, percentage of germination and seed vigour. The treatment of picking of 10 green fruits produced the highest green fruit yield/ha. But picking of first 4 green fruits produced maximum seed yield, which was statistically at par with those of 2 and 6 green fruits picking. First 4 green fruit picking gave the highest seed vigour whereas first 6 green fruit pickings produced maximum 100-seed weight. Picking of first 4 green fruits accompanied with $175 \mathrm{~kg} \mathrm{~N} / \mathrm{ha}$ gave the highest seed yield of 2.86 t/ha, which was closely followed by picking of first 2 or 6 green fruits with $125 \mathrm{~kg}$ as well as $175 \mathrm{~kg} \mathrm{~N} / \mathrm{ha}$. Picking of first 2 or 4 green fruits in combination with $125 \mathrm{~kg} \mathrm{~N} / \mathrm{ha}$ also gave the similar seed yield. The combination of $125 \mathrm{~kg} \mathrm{~N} / \mathrm{ha}$ and first 6 green fruit picking gave the highest gross income (Tk.193.56/ha), net return (Tk.126.35/ha) and maximum BCR of 2.88.
\end{abstract}

Key words: Nitrogen, picking, okra, fruit and seed yield, profitability.

\section{INTRODUCTION}

Okra (Abelmoschus esculentus (L.)Moench), commonly known as lady's finger, is a popular summer vegetable in Bangladesh. It originated in West Africa is an annual vegetable crop grown from seed in tropical and subtropical parts of the world (Thakur and Arora, 1986). It is a nutritious vegetable, which plays an important role to meet the demand of vegetables of the country when vegetables are scanty in the market (Ahmad, 1995). The green fruits are rich sources of vitamins, calcium, potassium and other minerals. In Bangladesh it is known as 'dherosh' which is also called

* Corresponding author: Senior Scientific Officer (Horticulture), ARS, Raikhali, BARI, Chandraghona, Bangladesh.

(C) 2009 School of Agriculture and Rural Development, Bangladesh Open University, All rights reserved. 
'bhindi' in India and Pakistan (Rashid, 1999). Judicious application of nitrogenous fertilizers has great impact on growth and yield of crop plants. The requirement of nitrogen which varies according to environmental conditions has to be determined by actual field trial for any particular soil and climate. Okra being a vegetable crop, its response to nitrogen should be high and higher dose of nitrogen is required to keep the fruits soft and edible. The recommend dose of nitrogen for the highest fruit yield appeared in various books and journals ranges from 120 to $200 \mathrm{~kg} / \mathrm{ha}$ (Amjad et al., 2001; Rahman et al, 1992; Paliwal et al., 1999; Verma and Batra 1999 ; Rashid, 1999) and for seed production ranges from 100 to $200 \mathrm{~kg} \mathrm{~N} / \mathrm{ha}$ (Birbal et al., 1995; Chattopadhyay and Sahana, 2000; Khan and Jaiswal 1988; Naik and Srinivas, 1992). There are reports that no difference in seed yield has been found if no picking of fresh fruit is done or only one or two pickings are made (Thakur and Arora, 1986). There are also reports that fruit setting in okra increases with a higher frequency of picking of fresh fruit (Natraj et al., 1992 ; Rashid 1999). Picking of green fruits encourages vegetative growth, fruit characters and ultimate seed yield and seed quality in okra (Singh et al., 1999; Khan and Jaiswal, 1988; Velumani and Ramaswamy, 1980). Seed crop is more profitable than fruit crop. As first some fruit pickings has no influence on seed production, picking green fruit plus seed yield give the best profit in okra cultivation. Such type of experimental results is not available in Bangladesh. In view of the above facts the present study was under taken to find out the optimum dose of nitrogen and number of green fruit picking on fruit as well as seed yield of okra for the highest economic benefit.

\section{MATERIALS AND Methods}

The experiment was conducted at the Agricultural Research Station, Raikhali in the district of Rangamati during the summer seasons of 2005 and 2006. This trial was carried out for both vegetable and seed production for higher benefit. The experimental field belongs to AEZ 29 with the Piedmont plain soil having medium loamy to moderately fine texture (sandy clay loam). The soil was poor in organic matter and moderately acidic. Nitrogen, Phosphorus, Potassium, Sulfur, Boron and Zinc were low in soil. N, P, K and Zn were below critical limit and B was as same as critical limit (Table 1). The experiment was laid out in a split-plot design with three replications, having four $\mathrm{N}$ levels $\left(\mathrm{N}_{0}=0, \mathrm{~N}_{1}=75, \mathrm{~N}_{2}=125\right.$ and $\left.\mathrm{N}_{3}=175 \mathrm{~kg} / \mathrm{ha}\right)$ as main plot treatments, and six levels of number of green fruit picking $\left(P_{0}=\right.$ no green fruit picking, $P_{1}=$ first 2 green fruit picking, $P_{2}=$ first 4 green fruit picking, $P_{3}=$ first 6 green fruit picking. $P_{4}=$ first 8 green fruit picking and $P_{5}=$ first 10 green fruits picking) being 2 fruits/picking as sub-plot treatments. There were 24 treatment combinations all together. The unit plot size was $4 \mathrm{~m} \times 2.4 \mathrm{~m}$. The initial soil test analyses were given in Table 1.

Table 1. Some physical and chemical properties of the soil of experimental plots prior to Sowing of crops

\begin{tabular}{|c|c|c|c|c|c|c|c|c|c|c|c|c|c|}
\hline \multirow{2}{*}{ Year } & \multirow{2}{*}{ Texture } & \multirow{2}{*}{$\mathrm{pH}$} & \multirow{2}{*}{$\begin{array}{c}\text { OM } \\
\%\end{array}$} & \multicolumn{3}{|c|}{ meq $100 \mathrm{~g}^{-1}$} & \multicolumn{7}{|c|}{$\mu q q^{-1}$} \\
\hline & & & & $\mathrm{Ca}$ & $\mathrm{Mg}$ & $\mathrm{K}$ & $\mathrm{NH} 4-\mathrm{N}$ & $\mathrm{P}$ & $\mathrm{S}$ & $\mathrm{B}$ & $\mathrm{Cu}$ & $\mathrm{Mn}$ & $\mathrm{Zn}$ \\
\hline 2005 & Sandy clay loam & 5.8 & 0.82 & 2.3 & 1.5 & 0.18 & 0.19 & 11 & 12 & 0.2 & 8 & 40 & 1.5 \\
\hline 2006 & Sandy clay loam & 5.7 & 0.88 & 2.5 & 1.6 & 0.18 & 0.18 & 10 & 12 & 0.2 & 7 & 35 & 1.4 \\
\hline Critical level & - & - & - & 2.0 & 0.8 & 0.2 & 75 & 14 & 14 & 0.2 & 1 & 33 & 2 \\
\hline
\end{tabular}

Manures and fertilizers were applied to the soil @ $100 \mathrm{~kg} \mathrm{P}_{2} \mathrm{O}_{5}, 150 \mathrm{~kg} \mathrm{~K}_{2} \mathrm{O}, 20 \mathrm{~kg} \mathrm{~S}, 1.0 \mathrm{~kg} \mathrm{~B}, 4.0$ $\mathrm{kg} \mathrm{Zn}, 1.0 \mathrm{~kg} \mathrm{Mo}$ and $\mathrm{N}$ as per treatment and 10 ton well rotten cowdung per hectare. The source of $\mathrm{N}, \mathrm{P}_{2} \mathrm{O}_{5}, \mathrm{~K} 2 \mathrm{O}, \mathrm{S}, \mathrm{B}, \mathrm{Zn}$ and Mo were Urea, TSP, MOP, gypsum, boric acid, $\mathrm{ZnO}$ and sodium molybdate. The total amount of cowdung, TSP, gypsum, boric acid, zinc and sodium molybdate and one-fourth quantity of urea and MOP were applied prior to planting seeds and the remaining three- fourth quantity of Urea and MP was top dressed into three equal installments 30,45 and 60 days after planting. Seeds of the variety BARI Dheros- 1 were dibbled at the rate of 2 seeds/ hill on 4 April in both 2005 and 2006 maintaining $60 \times 40 \mathrm{~cm}$ plant spacing. Prior to planting, seeds were soaked in tap water for 24 hours for uniform and better germination. After the germination was completed, thinning was done leaving one seedling in each hole. The harvesting of green fruit was 
started on 22 May, 2005 and 25 May, 2006 but the harvest of fruits for seed was started 7 June in both the years and continued up to end of October. All the recommended cultural and plant protection measures were followed throughout the experimental period.

The data on growth parameters, green fruit characters, dry fruit characters, green fruit yield and seed yield were recorded from randomly selected 10 plants from the inner rows of the plots. The plot yield from the harvest area of $9.6 \mathrm{~m}^{2}$ was calculated for both green fruit and seed and then it was converted to per hectare yield. For Percentage of germination and seed vigour index 100 fresh okra seed of each treatment was placed at trays with treated sands in the laboratory of ARS, Raikhali. Seed vigour index was calculated by using the formula suggested by Agarwal (1999) which is as follows-

Seed vigour index $=$

$\frac{\text { No. of germinated seeds (first count) }}{\text { Days to first count }}+\frac{\text { No. of germinated seeds (2nd count) }}{\text { Days to } 2 \text { nd count }}+\frac{\text { No. of germinated seeds (final count) }}{\text { Days to final count }}$

The treatment means were separated by DMRT at $5 \%$ level of significance for interpretation of the results.

\section{Results AND Discussion}

Since there were no significant differences between the results of the two years in respect of different parameters pooled analyses were done and discussed accordingly.

Green fruit yield of okra was increased with the increase of nitrogen from 0 to125 $\mathrm{kg} \mathrm{N} / \mathrm{ha}$ (Table 2). Significantly higher fruit yield was recorded under $125 \mathrm{~kg} \mathrm{~N} / \mathrm{ha}$ which was $12 \%$ higher than control. However, increase in nitrogen upto175 kg N/ha did not result into further increase in fruit yield over $125 \mathrm{~kg} \mathrm{~N} / \mathrm{ha}$. This is in agreement with Paliwal et al. (1999) and Rahman et al. (1992) but the result slightly differed with Talukder et al. (1999) and Verma and Batra (2001) who reported that application of $100 \mathrm{~kg}$ and $150 \mathrm{~kg} \mathrm{~N} / \mathrm{ha}$ respectively gave the highest yield of fruit. Successive increase in nitrogen levels up to $125 \mathrm{~kg} / \mathrm{ha}$ increased the fruit yield significantly over its lower levels. Nitrogen had linear response to seed yield from 0 to $125 \mathrm{~kg} \mathrm{~N} / \mathrm{ha}$ and further increase of nitrogen (175 kg N/ha) did not increase seed yield (Table 4). Significantly highest seed yield was recorded under $125 \mathrm{~kg} \mathrm{~N} / \mathrm{ha}$, which was $104 \%$ and $36.8 \%$ higher than control and $75 \mathrm{~kg} \mathrm{~N} / \mathrm{ha}$. This is in partial agreement with Khan and Jaiswal (1988) and Naik and Srinivas (1992) who got the highest seed yield of okra from 150 and $200 \mathrm{~kg} \mathrm{~N} / \mathrm{ha}$, respectively. In Bangladesh Mozumder et al. (2002) and Uddin et al. (2006) got the maximum seed yield of okra at 100 and $80 \mathrm{~kg} \mathrm{~N} / \mathrm{ha}$, respectively. Birbal et al. (1995) and Chattopadhyay and Sahana (2000) obtained the highest seed yield from the application of $100 \mathrm{~kg} \mathrm{~N} / \mathrm{ha}$ in India. Green fruit picking had linear increase of green fruit yield, the highest fruit yield being obtained from $P_{5}$ treatment (Table 2). This is in agreement with Bhuibhar et al. (1989) who received the highest yield of green fruit from the highest green fruit harvest (4 green fruit harvests). Isaac et al. (1998) also reported that green fruit picking improved fruit production in okra. Similar seed yield responses were noticed in $P_{0}, P_{1}, P_{2}$ and $P_{3}$ treatments and then seed yield decreased (Table 4). Velumani and Ramaswamy (1980) suggested harvesting 1 or 2 fresh fruits first formed without affecting seed yield and quality.

Nitrogen levels and green fruit picking combinedly put significant effect on plant height at first flowering, length of green fruit, fruit yield/plant, fruit yield/ha, number of dry fruits/plant, number of seeds/fruit, seed yield/plant and seed yield/ha but days to first flowering, days to first harvest of green fruit, diameter of green and dry fruit, weight of single green and dry fruit, weight of seeds/fruit, length of dry fruit did not show any significant interaction (Tables 3 and 5). The $\mathrm{N}_{2} \mathrm{P}_{4}$ combination gave maximum plant height at $1^{\text {st }}$ flowering $(50.33 \mathrm{~cm})$ which was at par with $\mathrm{N}_{2} \mathrm{P}_{3}$ and $\mathrm{N}_{2} \mathrm{P}_{5}$ combination (Table 3). Maximum length of green fruit was recorded in $\mathrm{N}_{2} \mathrm{P}_{5}(14.99 \mathrm{~cm})$ closely followed by $\mathrm{N}_{2} \mathrm{P}_{1}, \mathrm{~N}_{2} \mathrm{P} 2, \mathrm{~N}_{2} \mathrm{P}_{3}, \mathrm{~N}_{2} \mathrm{P}_{4}, \mathrm{~N}_{2} \mathrm{P}_{1}, \mathrm{~N}_{3} \mathrm{P}_{1}, \mathrm{~N}_{3} \mathrm{P}_{2}, \mathrm{~N}_{3} \mathrm{P}_{3}, \mathrm{~N}_{3} \mathrm{P}_{4}$ and $\mathrm{N}_{3} \mathrm{P}_{5}, \mathrm{~N}_{0} \mathrm{P}_{5}, \mathrm{~N}_{1} \mathrm{P}_{1}$ combinations. Maximum fruit yield per plant $(166.45 \mathrm{~g})$ as well as per hectare $(5.60 \mathrm{t})$ was obtained from $\mathrm{N}_{2} \mathrm{P}_{5}$ treatment followed by $\mathrm{N}_{3} \mathrm{P}_{5}$ treatment. 
Table 2. Effects of nitrogen and green fruit picking on growth, yield attributes and yield of green fruit of okra (pooled of 2005 and 2006)

\begin{tabular}{|c|c|c|c|c|c|c|c|c|}
\hline Treatment & $\begin{array}{l}\text { Days to } 1^{\text {st }} \\
\text { flowering }\end{array}$ & $\begin{array}{l}\text { Days to } 1^{\text {st }} \\
\text { harvest of } \\
\text { green fruit }\end{array}$ & $\begin{array}{l}\text { Plant height at } \\
1^{\text {st }} \text { flowering } \\
\text { (cm) }\end{array}$ & $\begin{array}{l}\text { Length of } \\
\text { green fruit } \\
\text { (cm) }\end{array}$ & $\begin{array}{l}\text { Diameter } \\
\text { of green } \\
\text { fruit }(\mathrm{cm})\end{array}$ & $\begin{array}{l}\text { Weight of } \\
\text { single green } \\
\text { fruit }(\mathrm{g})\end{array}$ & $\begin{array}{c}\text { Fruit } \\
\text { yield/ } \\
\text { plant (g) }\end{array}$ & $\begin{array}{l}\text { Fruit } \\
\text { yield } \\
\text { (t/ha) }\end{array}$ \\
\hline \multicolumn{9}{|l|}{ Nitrogen } \\
\hline $\mathrm{N}_{0}$ & 30.72 & 38.57 & $35.55 c$ & $11.26 \mathrm{~b}$ & 1.25 & $11.02 \mathrm{~b}$ & $68.91 \mathrm{c}$ & $2.44 \mathrm{~b}$ \\
\hline $\mathrm{N}_{1}$ & 31.67 & 39.50 & $36.78 c$ & $11.33 b$ & 1.24 & 11.66ab & 72.33b & $2.48 \mathrm{~b}$ \\
\hline $\mathrm{N}_{2}$ & 31.09 & 39.26 & $47.04 a$ & $12.19 a$ & 1.25 & $12.33 a$ & $78.43 a$ & $2.74 a$ \\
\hline $\mathrm{N}_{3}$ & 31.61 & 39.39 & $44.67 \mathrm{~b}$ & 11.99ab & 1.24 & $11.98 a$ & $72.30 \mathrm{~b}$ & $2.64 a$ \\
\hline \multirow{2}{*}{\multicolumn{9}{|c|}{$\begin{array}{l}\text { CV (\%) } \\
\text { Green fruit picking }\end{array}$}} \\
\hline & & & & & & & & \\
\hline $\mathrm{P}_{0}$ & 31.38 & - & 39.63 & - & - & - & - & - \\
\hline$P_{1}$ & 31.45 & 38.86 & 40.04 & $13.70 \mathrm{~b}$ & $1.42 \mathrm{~b}$ & 13.73 & $23.68 d$ & $1.04 \mathrm{e}$ \\
\hline $\mathrm{P}_{2}$ & 31.14 & 38.91 & 39.68 & $13.78 b$ & $1.42 \mathrm{~b}$ & 13.91 & $57.10 c$ & $2.08 d$ \\
\hline $\mathrm{P}_{3}$ & 31.50 & 38.45 & 39.92 & $13.94 \mathrm{~b}$ & $1.48 \mathrm{a}$ & 14.08 & $88.21 d$ & $3.00 c$ \\
\hline $\mathrm{P}_{4}$ & 31.12 & 38.49 & 40.68 & $13.93 \mathrm{~b}$ & $1.48 \mathrm{a}$ & 14.28 & $116.68 \mathrm{~b}$ & $4.27 \mathrm{~b}$ \\
\hline$P_{5}$ & 31.05 & 38.16 & 40.36 & $14.43 a$ & $1.49 a$ & 14.38 & $151.65 a$ & $5.28 a$ \\
\hline$\overline{C V}(\%)$ & 4.30 & 6.21 & 6.01 & 4.61 & 4.61 & 6.31 & 4.40 & 6.60 \\
\hline
\end{tabular}

Means having same letters or without letter in a column are not significantly different at $5 \%$ level of probability by DMRT $\mathrm{N}=$ Nitrogen, $\mathrm{P}=$ Green fruit picking

$\mathrm{N}_{0}=0 \mathrm{~kg} / \mathrm{a}, \mathrm{N}_{1}=75 \mathrm{~kg} / \mathrm{ha}, \mathrm{N}_{2}=125 \mathrm{~kg} / \mathrm{ha}, \mathrm{N}_{3}=175 \mathrm{~kg} / \mathrm{ha}$

$\mathrm{P}_{0}=$ No green fruit harvest, $\mathrm{P}_{1}=$ First two green fruit harvest, $\mathrm{P}_{2}=$ First four green fruit harvest,

$P_{3}=$ First six green fruit harvest, $P_{4}=$ First eight green fruit harvest, $P_{5}=$ First 10 green fruit harvest

Table 3. Combined effect of nitrogen and green fruit picking on growth, yield attributes and yield of green fruit of okra (pooled of 2005 and 2006)

\begin{tabular}{|c|c|c|c|c|c|c|c|c|c|}
\hline \multicolumn{2}{|c|}{$\begin{array}{c}\text { Treatment } \\
\text { combination }\end{array}$} & \multirow{2}{*}{$\begin{array}{l}\text { Days to } 1^{\text {st }} \\
\text { flowering }\end{array}$} & \multirow{2}{*}{$\begin{array}{l}\text { Days to } 1^{\text {st }} \\
\text { harvest of } \\
\text { green fruit }\end{array}$} & \multirow{2}{*}{$\begin{array}{l}\text { Plant height a } \\
1^{\text {st }} \text { flowering } \\
\text { (cm) }\end{array}$} & \multirow{2}{*}{$\begin{array}{l}\text { Length of } \\
\text { green } \\
\text { fruit }(\mathrm{cm})\end{array}$} & \multirow{2}{*}{$\begin{array}{l}\text { Diameter } \\
\text { of green } \\
\text { fruit }(\mathrm{cm})\end{array}$} & \multirow{2}{*}{$\begin{array}{l}\text { Weight of } \\
\text { single green } \\
\text { fruit weight }(\mathrm{g})\end{array}$} & \multirow{2}{*}{$\begin{array}{c}\text { Fruit } \\
\text { yield/ } \\
\text { plant }(\mathrm{g})\end{array}$} & \multirow{2}{*}{$\begin{array}{l}\text { Fruit } \\
\text { yield } \\
\text { (t/ha) }\end{array}$} \\
\hline $\mathrm{N}$ & $\mathrm{P}$ & & & & & & & & \\
\hline \multirow{6}{*}{$\mathrm{N}_{0}$} & $\mathrm{P}_{0}$ & 30.09 & - & $35.30 \mathrm{gh}$ & - & - & - & - & - \\
\hline & $\mathrm{P}_{1}$ & 29.82 & 36.94 & $36.24 \mathrm{gh}$ & $12.69 f$ & 1.40 & 12.22 & $22.92 \mathrm{k}$ & $0.89 \mathrm{i}$ \\
\hline & $\mathrm{P}_{2}$ & 31.79 & 38.10 & $35.37 \mathrm{gh}$ & $13.45 \mathrm{ef}$ & 1.45 & 12.60 & $52.80 \mathrm{j}$ & $1.84 \mathrm{~h}$ \\
\hline & $P_{3}$ & 30.90 & 38.60 & $34.60 \mathrm{gh}$ & 13.71cde & 1.57 & 13.92 & $84.71 \mathrm{~h}$ & $2.98 \mathrm{e}$ \\
\hline & $\mathrm{P}_{4}$ & 30.17 & 36.23 & $35.30 \mathrm{gh}$ & 13.51def & 1.52 & 13.49 & $109.48 f$ & $3.93 d$ \\
\hline & $P_{5}$ & 31.54 & 37.38 & $33.69 \mathrm{~h}$ & $14.56 a-d$ & 1.60 & 14.55 & $145.54 \mathrm{c}$ & $4.09 \mathrm{~b}$ \\
\hline \multirow{6}{*}{$\mathrm{N}_{1}$} & $\mathrm{P}_{0}$ & 31.58 & - & $36.27 \mathrm{gh}$ & 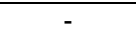 & 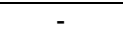 & - & - & 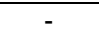 \\
\hline & $\mathrm{P}_{1}$ & 32.35 & 39.44 & $36.10 \mathrm{gh}$ & 13.99a-e & 1.46 & 14.40 & $24.05 \mathrm{k}$ & $1.06 \mathrm{i}$ \\
\hline & $\mathrm{P}_{2}$ & 30.23 & 38.75 & $33.70 \mathrm{~h}$ & 13.54def & 1.44 & 14.26 & $55.50 \mathrm{ij}$ & $2.05 \mathrm{gh}$ \\
\hline & $P_{3}$ & 32.86 & 38.72 & $35.20 \mathrm{gh}$ & $13.26 \mathrm{ef}$ & 1.51 & 13.75 & $88.88 \mathrm{~h}$ & $2.57 f$ \\
\hline & $\mathrm{P}_{4}$ & 30.80 & 38.37 & $38.38 \mathrm{fg}$ & $13.75 b-e$ & 1.53 & 14.52 & $114.95 \mathrm{e}$ & $4.24 c$ \\
\hline & $\mathrm{P}_{5}$ & 32.39 & 38.94 & $38.25 f g$ & $13.78 \mathrm{~b}-\mathrm{e}$ & 1.48 & 13.72 & $152.71 b$ & $5.04 \mathrm{~b}$ \\
\hline \multirow{6}{*}{$\mathrm{N}_{2}$} & $\mathrm{P}_{0}$ & 31.88 & & $43.88 \mathrm{~cd}$ & 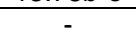 & 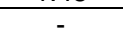 & 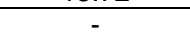 & $\cdots$ & 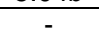 \\
\hline & $P_{1}$ & 31.41 & 39.06 & 42.21cde & 14.18a-e & 1.47 & 14.23 & $26.21 \mathrm{k}$ & $1.05 \mathrm{i}$ \\
\hline & $\mathrm{P}_{2}$ & 31.03 & 39.07 & $44.32 \mathrm{~cd}$ & $14.55 a-d$ & 1.45 & 15.11 & $60.50 \mathrm{i}$ & $2.21 \mathrm{~g}$ \\
\hline & $P_{3}$ & 31.64 & 38.33 & $49.40 a$ & $14.98 a$ & 1.58 & 15.03 & $96.88 \mathrm{~g}$ & $3.27 \mathrm{e}$ \\
\hline & $\mathrm{P}_{4}$ & 31.31 & 39.02 & $50.33 a$ & 14.78ab & 1.53 & 14.92 & $125.24 d$ & $4.39 c$ \\
\hline & $P_{5}$ & 29.29 & 37.48 & $48.42 \mathrm{ab}$ & $14.99 a$ & 1.56 & 15.41 & $166.45 a$ & $5.60 \mathrm{a}$ \\
\hline \multirow{6}{*}{$\mathrm{N}_{3}$} & $\mathrm{P}_{0}$ & 31.98 & & 41.28def & - & - & & - & - \\
\hline & $\mathrm{P}_{1}$ & 32.22 & 39.55 & $41.25 \mathrm{def}$ & $14.22 \mathrm{a}-\mathrm{e}$ & 1.46 & 14.60 & $22.76 \mathrm{k}$ & $1.14 \mathrm{i}$ \\
\hline & $P_{2}$ & 31.49 & 39.50 & $43.96 \mathrm{~cd}$ & 13.94a-e & 1.46 & 14.26 & $52.57 \mathrm{j}$ & $2.14 \mathrm{~g}$ \\
\hline & $P_{3}$ & 30.97 & 37.71 & 40.05ef & 14.12a-e & 1.47 & 14.12 & $84.18 \mathrm{~h}$ & $3.14 \mathrm{e}$ \\
\hline & $\mathrm{P}_{4}$ & 32.19 & 39.56 & 40.98def & 13.98a-e & 1.56 & 15.08 & $119.28 \mathrm{e}$ & $4.39 c$ \\
\hline & $P_{5}$ & 30.85 & 37.26 & $45.26 \mathrm{bc}$ & $14.64 \mathrm{abc}$ & 1.53 & 13.51 & $154.93 b$ & $5.15 b$ \\
\hline CV (\%) & & 4.30 & 6.21 & 6.01 & 4.61 & 4.79 & 6.31 & 4.40 & 6.60 \\
\hline
\end{tabular}

Means having same letter or without letter in a column are not significantly different at $5 \%$ level of probability by DMRT

$\mathrm{N}=$ Nitrogen, $\mathrm{P}=$ Green fruit picking

$N_{0}=0 \mathrm{~kg} / \mathrm{a}, N_{1}=75 \mathrm{~kg} / \mathrm{ha}, \mathrm{N}_{2}=125 \mathrm{~kg} / \mathrm{ha}, \mathrm{N}_{3}=175 \mathrm{~kg} / \mathrm{ha}$

$P_{0}=$ No green fruit harvest, $P_{1}=$ First two green fruit harvest, $P_{2}=$ First four green fruit harvest,

$P_{3}=$ First six green fruit harvest, $P_{4}=$ First eight green fruit harvest, $P_{5}=$ First 10 green fruit harvest 
Effect of Nitrogen Levels and Picking of Green Fruits on the Fruit and Seed Production of Okra

Table 4. Effect of nitrogen and green fruit picking on yield attributes and seed yield of okra (pooled of 2005 and 2006)

\begin{tabular}{lcccccccc}
\hline Treatment & $\begin{array}{c}\text { Dry fruits } \\
\text { (no.) }\end{array}$ & $\begin{array}{c}\text { Weight of } \\
\text { single dry fruit } \\
\text { weight (g) }\end{array}$ & $\begin{array}{c}\text { Seeds/ } \\
\text { fruit (no.) }\end{array}$ & $\begin{array}{c}\text { Weight of } \\
\text { sfeeds } \\
\text { ffruit (g) }\end{array}$ & $\begin{array}{c}\text { Length of } \\
\text { dry fruit } \\
\text { (cm) }\end{array}$ & $\begin{array}{c}\text { Diameter } \\
\text { of dry fruit } \\
\text { (cm) }\end{array}$ & $\begin{array}{c}\text { Seed } \\
\text { yield/ } \\
\text { plant (g) }\end{array}$ & $\begin{array}{c}\text { Seed } \\
\text { yield } \\
\text { (t/ha) }\end{array}$ \\
\hline Nitrogen & & & & & & & & \\
$\mathrm{N}_{0}$ & $17.60 \mathrm{~d}$ & 5.72 & $33.95 \mathrm{~d}$ & $1.96 \mathrm{~d}$ & $15.98 \mathrm{c}$ & 1.50 & $36.73 \mathrm{c}$ & $1.24 \mathrm{c}$ \\
$\mathrm{N}_{1}$ & $23.03 \mathrm{c}$ & 5.58 & $37.68 \mathrm{c}$ & $2.27 \mathrm{~b}$ & $17.75 \mathrm{~b}$ & 1.51 & $52.13 \mathrm{~b}$ & $1.85 \mathrm{~b}$ \\
$\mathrm{~N}_{2}$ & $26.98 \mathrm{a}$ & 6.12 & $42.48 \mathrm{~b}$ & $2.60 \mathrm{a}$ & $18.27 \mathrm{a}$ & 1.53 & $69.95 \mathrm{a}$ & $2.53 \mathrm{a}$ \\
$\mathrm{N}_{3}$ & $26.14 \mathrm{~b}$ & 5.94 & $43.30 \mathrm{a}$ & $2.66 \mathrm{a}$ & $18.63 \mathrm{a}$ & 1.52 & $69.75 \mathrm{a}$ & $2.54 \mathrm{a}$ \\
Green fruit picking & & & & & & & & \\
$\mathrm{P}_{0}$ & $24.25 \mathrm{~d}$ & 5.69 & $39.49 \mathrm{~b}$ & $2.47 \mathrm{a}$ & 17.62 & 1.51 & $58.88 \mathrm{~b}$ & $2.15 \mathrm{~b}$ \\
$\mathrm{P}_{1}$ & $24.73 \mathrm{c}$ & 5.74 & $39.89 \mathrm{a}$ & $2.42 \mathrm{a}$ & 17.44 & 1.48 & $60.75 \mathrm{ab}$ & $2.20 \mathrm{ab}$ \\
$\mathrm{P}_{2}$ & $24.77 \mathrm{~b}$ & 6.03 & $39.75 \mathrm{a}$ & $2.42 \mathrm{a}$ & 17.72 & 1.53 & $61.93 \mathrm{ab}$ & $2.26 \mathrm{a}$ \\
$\mathrm{P}_{3}$ & $25.47 \mathrm{a}$ & 5.58 & $39.30 \mathrm{c}$ & $2.40 \mathrm{~b}$ & 17.52 & 1.51 & $62.68 \mathrm{a}$ & $2.25 \mathrm{a}$ \\
$\mathrm{P}_{4}$ & $21.93 \mathrm{e}$ & 5.86 & $38.91 \mathrm{~d}$ & $2.33 \mathrm{~d}$ & 17.91 & 1.52 & $51.90 \mathrm{c}$ & $1.88 \mathrm{c}$ \\
$\mathrm{P}_{5}$ & $18.65 \mathrm{f}$ & 5.93 & $38.86 \mathrm{~d}$ & $2.30 \mathrm{e}$ & 17.69 & 1.53 & $43.71 \mathrm{~d}$ & $1.60 \mathrm{c}$ \\
\hline $\mathrm{CV}(\%)$ & 5.01 & 10.55 & 4.53 & 3.91 & 4.57 & 4.61 & 6.33 & 6.30 \\
\hline
\end{tabular}

Means having same letter or without letter in a column are not significantly different at $5 \%$ level of probability DMRT

$\mathrm{N}=$ Nitrogen, $\mathrm{P}=$ Green fruit picking

$N_{0}=0 \mathrm{~kg} / \mathrm{a}, \mathrm{N}_{1}=75 \mathrm{~kg} / \mathrm{ha}, \mathrm{N}_{2}=125 \mathrm{~kg} / \mathrm{ha}, \mathrm{N}_{3}=175 \mathrm{~kg} / \mathrm{ha}$

$P_{0}=$ No green fruit harvest, $P_{1}=$ First two green fruit harvest, $P_{2}=$ First four green fruit harvest,

$P_{3}=$ First six green fruit harvest, $P_{4}=$ First eight green fruit harvest, $P_{5}=$ First 10 green fruit harvest

Table 5. Combined effect of nitrogen and green fruit picking on yield attributes and seed yield of okra pooled of 2005 and 2006)

\begin{tabular}{|c|c|c|c|c|c|c|c|c|c|}
\hline \multicolumn{2}{|c|}{$\begin{array}{c}\text { Treatment } \\
\text { combination }\end{array}$} & \multirow{2}{*}{$\begin{array}{l}\text { Dry fruits } \\
\text { (no.) }\end{array}$} & \multirow{2}{*}{$\begin{array}{c}\text { Weight of } \\
\text { single dry fruit } \\
\text { weight }(\mathrm{g})\end{array}$} & \multirow{2}{*}{$\begin{array}{l}\text { Seeds/ } \\
\text { fruit (no.) }\end{array}$} & \multirow{2}{*}{$\begin{array}{l}\text { Weight of } \\
\text { seeds } \\
\text { /fruit (g) }\end{array}$} & \multirow{2}{*}{$\begin{array}{l}\text { Length of } \\
\text { dry fruit } \\
(\mathrm{cm})\end{array}$} & \multirow{2}{*}{$\begin{array}{l}\text { Diameter } \\
\text { of dry fruit } \\
(\mathrm{cm})\end{array}$} & \multirow{2}{*}{$\begin{array}{l}\text { Seed yield/ } \\
\text { plant (g) }\end{array}$} & \multirow{2}{*}{$\begin{array}{l}\text { Seed yield } \\
\text { (t/ha) }\end{array}$} \\
\hline $\mathrm{N}$ & $\mathrm{P}$ & & & & & & & & \\
\hline \multirow{6}{*}{$\mathrm{N}_{0}$} & $P_{0}$ & $|18.14|$ & 5.85 & $34.19 \mathrm{~lm}$ & 1.99 & 16.10 & 1.55 & $35.66 \mathrm{ef}$ & $1.29 f$ \\
\hline & $P_{1}$ & $18.54 \mid$ & 5.72 & $34.52 \mid$ & 2.05 & 15.94 & 1.49 & $37.33 \mathrm{ef}$ & $1.34 f$ \\
\hline & $\mathrm{P}_{2}$ & $18.92 \mathrm{kl}$ & 5.94 & $34.67 \mathrm{Im}$ & 2.05 & 16.18 & 1.56 & $38.13 e$ & $1.39 f$ \\
\hline & $\mathrm{P}_{3}$ & $19.46 \mathrm{k}$ & 5.78 & $34.15 \mathrm{~m}$ & 2.02 & 15.99 & 1.55 & $38.76 \mathrm{e}$ & $1.39 f$ \\
\hline & $\mathrm{P}_{4}$ & $16.47 \mathrm{~m}$ & 5.49 & 33.50n & 1.93 & 16.21 & 1.55 & $31.34 \mathrm{fg}$ & $1.02 \mathrm{~g}$ \\
\hline & $P_{5}$ & $14.05 n$ & 5.71 & $33.50 n$ & 1.91 & 15.98 & 1.51 & $27.03 \mathrm{~g}$ & $0.98 \mathrm{~g}$ \\
\hline \multirow{6}{*}{$\mathrm{N}_{1}$} & $P_{0}$ & $23.99 \mathrm{i}$ & 5.55 & $38.00 \mathrm{j}$ & 2.30 & 17.89 & 1.54 & $54.20 \mathrm{c}$ & $1.95 \mathrm{~d}$ \\
\hline & $P_{1}$ & 24.47hi & 5.88 & $38.37 i$ & 2.35 & 17.71 & 1.48 & $57.26 \mathrm{c}$ & $2.00 d$ \\
\hline & $\mathrm{P}_{2}$ & $24.96 \mathrm{gh}$ & 5.89 & 38.20ij & 2.33 & 17.97 & 1.54 & $57.95 \mathrm{c}$ & $2.03 d$ \\
\hline & $\mathrm{P}_{3}$ & $24.94 \mathrm{gh}$ & 5.35 & $37.95 \mathrm{j}$ & 2.34 & 17.77 & 1.46 & $57.38 \mathrm{c}$ & $2.07 d$ \\
\hline & $\mathrm{P}_{4}$ & $21.41 \mathrm{j}$ & 5.50 & $37.23 \mathrm{k}$ & 2.24 & 17.99 & 1.47 & $47.20 d$ & $1.67 \mathrm{e}$ \\
\hline & $P_{5}$ & 18.201 & 5.30 & $37.24 \mathrm{k}$ & 2.22 & 17.74 & 1.51 & $39.74 \mathrm{e}$ & $1.46 f$ \\
\hline \multirow{6}{*}{$\mathrm{N}_{2}$} & $\mathrm{P}_{0}$ & $27.83 \mathrm{de}$ & 5.53 & $42.90 f$ & 2.64 & 18.34 & 1.53 & $73.04 a$ & $2.69 a b$ \\
\hline & $P_{1}$ & $28.39 \mathrm{bcd}$ & 6.15 & $43.12 \mathrm{de}$ & 2.68 & 18.16 & 1.52 & $74.90 \mathrm{a}$ & $2.73 a b$ \\
\hline & $\mathrm{P}_{2}$ & $28.95 b$ & 6.32 & 42.93ef & 2.67 & 18.42 & 1.52 & $76.19 a$ & $2.75 a b$ \\
\hline & $\mathrm{P}_{3}$ & $29.82 a$ & 5.74 & $42.67 f$ & 2.66 & 18.22 & 1.52 & $77.80 \mathrm{a}$ & $2.79 a b$ \\
\hline & $\mathrm{P}_{4}$ & $25.35 \mathrm{~g}$ & 6.80 & $42.25 \mathrm{~g}$ & 2.61 & 18.45 & 1.54 & $64.86 \mathrm{~b}$ & $2.40 \mathrm{c}$ \\
\hline & $P_{5}$ & $21.14 j$ & 6.46 & $41.84 \mathrm{~h}$ & 2.55 & 18.29 & 1.56 & $53.90 c$ & $1.99 d$ \\
\hline \multirow{6}{*}{$\mathrm{N}_{3}$} & $\mathrm{P}_{0}$ & $27.01 \mathrm{f}$ & 5.87 & $43.57 \mathrm{bc}$ & 2.70 & 18.52 & 1.49 & $73.59 a$ & $2.67 a b$ \\
\hline & $P_{1}$ & $27.55 f$ & 5.38 & $44.00 \mathrm{a}$ & 2.74 & 18.34 & 1.46 & $74.35 a$ & $2.71 a b$ \\
\hline & $\mathrm{P}_{2}$ & $28.09 \mathrm{cde}$ & 6.13 & 43.84ab & 2.75 & 18.67 & 1.55 & $76.02 a$ & $2.86 a$ \\
\hline & $\mathrm{P}_{3}$ & $28.84 b c$ & 5.82 & 42.98def & 2.69 & 18.47 & 1.53 & $76.18 a$ & $2.78 a b$ \\
\hline & $\mathrm{P}_{4}$ & $24.51 \mathrm{hi}$ & 5.98 & 43.13de & 2.70 & 19.39 & 1.53 & $64.20 \mathrm{~b}$ & $2.35 c$ \\
\hline & $P_{5}$ & $20.83 j$ & 6.40 & $43.55 \mathrm{~cd}$ & 2.65 & 19.12 & 1.54 & $54.15 \mathrm{c}$ & $1.99 d$ \\
\hline \multicolumn{2}{|c|}{ CV (\%) } & 5.01 & 10.55 & 4.53 & 3.91 & 4.57 & 4.61 & 6.33 & 6.30 \\
\hline
\end{tabular}

Means having same letter or without letter in a column are not significantly different at $5 \%$ level of probability by DMRT $\mathrm{N}=$ Nitrogen, $\mathrm{P}=$ Green fruit picking

$\mathrm{N}_{0}=0 \mathrm{~kg} / \mathrm{a}, \mathrm{N}_{1}=75 \mathrm{~kg} / \mathrm{ha}, \mathrm{N}_{2}=125 \mathrm{~kg} / \mathrm{ha}, \mathrm{N}_{3}=175 \mathrm{~kg} / \mathrm{ha}$

$P_{0}=$ No green fruit harvest, $P_{1}=$ First two green fruit harvest, $P_{2}=$ First four green fruit harvest,

$P_{3}=$ First six green fruit harvest, $P_{4}=$ First eight green fruit harvest, $P_{5}=$ First 10 green fruit harvest 
Maximum number of dry fruits/plant was obtained from $\mathrm{N}_{2} \mathrm{P}_{3}$ (29.82) significantly followed by $\mathrm{N}_{2} \mathrm{P}_{3}$ (28.84) and the lowest from $\mathrm{N}_{0} \mathrm{P}_{0}$. Maximum number of seeds/fruit was recorded in $\mathrm{N}_{3} \mathrm{P}_{1}(44.00)$ that were statistically similar to $\mathrm{N}_{3} \mathrm{P}_{2}$ (Table 5). The highest seed yield/plant was obtained from $\mathrm{N}_{2} \mathrm{P}_{3}$ (77.80 g) closely followed by $\mathrm{N}_{1} \mathrm{P}_{0}, \mathrm{~N}_{2} \mathrm{P}_{0}, \quad \mathrm{~N}_{2} \mathrm{P}_{1}, \mathrm{~N}_{2} \mathrm{P}_{2}, \mathrm{~N}_{3} \mathrm{P}_{0}, \mathrm{~N}_{3} \mathrm{P}_{1}, \mathrm{~N}_{3} \mathrm{P}_{2}$ and $\mathrm{N}_{3} \mathrm{P}_{3}$ combinations and the lowest seed yield/plant from $\mathrm{N}_{0} \mathrm{P}_{0}$ combination. The $\mathrm{N}_{3} \mathrm{P}_{2}$ combination produced the maximum seed yield (2.86 t/ha) which was statistically similar to $N_{1} P_{0}, N_{2} P_{1}, N_{2} P_{2}$, $\mathrm{N}_{2} \mathrm{P}_{3}, \mathrm{~N}_{3} \mathrm{P}_{0}, \mathrm{~N}_{3} \mathrm{P}_{1}$ and $\mathrm{N}_{3} \mathrm{P}_{3}$ combinations. Natraj et al. (1992) found no adverse effect on seed yield and quality by picking 4 fruits/plant and applying 125 or $175 \mathrm{~kg} \mathrm{~N} / \mathrm{ha}$.

Quality attributes of seed were shown in Table 6. Maximum 100-seed weight (61.39), germination percentage (95.47\%) and seed vigour (41.2\%) were obtained when N was applied @ $175 \mathrm{~kg} / \mathrm{ha}$. Application of N@125 kg/ha gave identical results to $175 \mathrm{~kg} \mathrm{~N} / / \mathrm{ha}$ in respect of $100-$ seed weight, germination percentage and seed vigour. The poorer results regarding 100 -seed weight, germination percentage and seed vigour were obtained when no nitrogen was applied (Table 6). These are in agreement with the report of Uddin et al. (2006) who got the highest germination percentage, seed vigour and 100-seed weight from $80 \mathrm{~kg} \mathrm{~N}$ application among 0,40 , 80 and $120 \mathrm{~kg} \mathrm{~N} / \mathrm{ha} .100$-seed weight and seed vigour except percentage of germination were significantly affected by green fruit picking (Table 6). The treatment $\mathrm{P}_{3}$ gave maximum 100 -seed weight $(60.82 \mathrm{~g})$ whereas $\mathrm{P}_{2}$ produced maximum seed vigour (40.82). But there were no significant differences among $P_{0}, P_{1}, P_{2}$ and $P_{3}$ treatments in terms of 100 -seed weight and seed vigour.

\section{Table 6. Effect of nitrogen and green fruit picking on seed quality of okra pooled} of 2005 and 2006)

\begin{tabular}{lccc}
\hline Treatment & 100-seed weight (g) & Germination (\%) & Seed vigour index \\
\hline Nitrogen & $57.77 \mathrm{c}$ & $91.98 \mathrm{c}$ & \\
$\mathrm{N}_{0}$ & $60.06 \mathrm{~b}$ & $94.95 \mathrm{~b}$ & $35.76 \mathrm{c}$ \\
$\mathrm{N}_{1}$ & $61.23 \mathrm{a}$ & $95.32 \mathrm{a}$ & $38.65 \mathrm{~b}$ \\
$\mathrm{~N}_{2}$ & $61.39 \mathrm{a}$ & $95.47 \mathrm{a}$ & $40.49 \mathrm{a}$ \\
$\mathrm{N}_{3}$ & & & $41.20 \mathrm{a}$ \\
Green fruit picking & $60.45 \mathrm{a}$ & 94.02 & $40.54 \mathrm{a}$ \\
$\mathrm{P}_{0}$ & $60.53 \mathrm{a}$ & 94.82 & $40.45 \mathrm{a}$ \\
$\mathrm{P}_{1}$ & $60.79 \mathrm{a}$ & 94.94 & $40.82 \mathrm{a}$ \\
$\mathrm{P}_{2}$ & $60.82 \mathrm{a}$ & 94.59 & $40.35 \mathrm{a}$ \\
$\mathrm{P}_{3}$ & $59.57 \mathrm{~b}$ & 94.94 & $38.37 \mathrm{~b}$ \\
$\mathrm{P}_{4}$ & $58.67 \mathrm{~b}$ & 94.90 & $36.14 \mathrm{c}$ \\
$\mathrm{P}_{5}$ & $\mathrm{~ns}$ & $\mathrm{~ns}$ & $\mathrm{~ns}$ \\
\hline $\mathrm{N} \wedge$ & 5.25 & 4.87 & 4.11 \\
\hline $\mathrm{CV}(\%)$ & & & \\
\hline
\end{tabular}

Means having same letter or without letter in a column are not significantly different at $5 \%$ level of probability by DMRT $\mathrm{N}=$ Nitrogen, $\mathrm{P}=$ Green fruit picking

$\mathrm{N}_{0}=0 \mathrm{~kg} / \mathrm{a}, \mathrm{N}_{1}=75 \mathrm{~kg} / \mathrm{ha}, \mathrm{N}_{2}=125 \mathrm{~kg} / \mathrm{ha}, \mathrm{N}_{3}=175 \mathrm{~kg} / \mathrm{ha}$

$\mathrm{P}_{0}=$ No green fruit harvest, $\mathrm{P}_{1}=$ First two green fruit harvest, $\mathrm{P}_{2}=$ First four green fruit harvest,

$P_{3}=$ First six green fruit harvest, $P_{4}=$ First eight green fruit harvest, $P_{5}=$ First 10 green fruit harvest

The cost-return analysis of okra production was given in Table 7. It revealed that maximum gross return from fruit was recorded in $\mathrm{N}_{2} \mathrm{P}_{5}$ combination whereas gross return from seed, recorded in $\mathrm{N}_{3} \mathrm{P}_{2}$ combination. The Table 5 also described that gross return of seed obtained from $\mathrm{N}_{2} \mathrm{P}_{2}$, $\mathrm{N}_{2} \mathrm{P}_{3}$ and $\mathrm{N}_{3} \mathrm{P}_{3}$ were very close to the $\mathrm{N}_{3} \mathrm{P}_{2}$ combination. The total gross return (Tk.193.56 thousand/ha) and net profit (Tk.126.35 thousand/ha) were found from $\mathrm{N}_{2} \mathrm{P}_{3}$ combination followed by $\mathrm{N}_{3} \mathrm{P}_{3}$ and $\mathrm{N}_{3} \mathrm{P}_{2}$ combination. The combinations of $\mathrm{N}_{2} \mathrm{P}_{3}, \mathrm{~N}_{3} \mathrm{P}_{3}$ and $\mathrm{N}_{2} \mathrm{P}_{2}$ also gave maximum $\mathrm{BCR}$ of 2.88, 2.84 and 2.79, respectively. This is in agreement with Bhuibhar et al. (1989) who obtained the highest economic benefit from picking of 4 green fruits per plant. Singh et al. (1999) also reported that green fruit picking increased seed yield compared with intact control. The combinations of $\mathrm{N}_{2} \mathrm{P}_{0}$ and $\mathrm{N}_{3} \mathrm{P}_{0}$ gave total gross return of Tk. 161.40 thousand/ha and Tk. 160.20 thousand/ha, respectively; these two combinations gave BCR of 2.57 and 2.53. From the Table 7, it was also 
clear that application of $125 \mathrm{~kg} \mathrm{~N} /$ ha produced maximum total gross return, net return and BCR irrespective of green fruit picking and first six green fruit picking $\left(P_{3}\right)$ also gave maximum total gross return, net return and $\mathrm{BCR}$ irrespective of $\mathrm{N}$ application.

Table 7. Cost-return analysis of okra production (average of two years)

\begin{tabular}{|c|c|c|c|c|c|c|c|c|}
\hline $\begin{array}{c}\text { Nitrogen } \\
\mathrm{X} \\
\text { Picking }\end{array}$ & $\begin{array}{c}\text { Yield of } \\
\text { green fruit } \\
\text { (t/ha) }\end{array}$ & $\begin{array}{c}\text { Gross } \\
\text { return from } \\
\text { fruit } \\
\text { (000Tk.) }\end{array}$ & $\begin{array}{c}\text { Yield of } \\
\text { seed (t/ha }\end{array}$ & $\begin{array}{c}\text { Gross } \\
\text { return from } \\
\text { seed } \\
\text { (000Tk.) }\end{array}$ & $\begin{array}{c}\text { Total gross } \\
\text { return (000 } \\
\text { Tk.) }\end{array}$ & $\begin{array}{l}\text { Cost of } \\
\text { production } \\
\text { (000 Tk.) }\end{array}$ & $\begin{array}{l}\text { Net profit } \\
(000 \text { Tk.) }\end{array}$ & $\begin{array}{c}\text { Benefit- } \\
\text { cost ratio } \\
\text { (BCR) }\end{array}$ \\
\hline $\mathrm{N}_{0} \mathrm{P}_{0}{ }^{*}$ & - & - & 1.29 & 77.40 & 77.40 & 60.20 & 17.20 & 1.28 \\
\hline $\mathrm{N}_{0} \mathrm{P}_{1}$ & 0.89 & 7.71 & 1.34 & 80.40 & 87.52 & 64.61 & 22.91 & 1.35 \\
\hline $\mathrm{N}_{0} \mathrm{P}_{2}$ & 1.84 & 14.72 & 1.39 & 83.40 & 98.12 & 64.47 & 33.65 & 1.52 \\
\hline $\mathrm{N}_{0} \mathrm{P}_{3}$ & 2.98 & 23.84 & 1.39 & 83.40 & 107.24 & 64.61 & 42.63 & 1.66 \\
\hline $\mathrm{N}_{0} \mathrm{P}_{4}$ & 3.93 & 31.44 & 1.02 & 61.20 & 92.64 & 64.68 & 27.96 & 1.43 \\
\hline $\mathrm{N}_{0} \mathrm{P}_{5}$ & 4.09 & 32.72 & 0.98 & 58.80 & 91.52 & 64.68 & 26.84 & 1.41 \\
\hline $\mathrm{N}_{1} \mathrm{P}_{0}$ & - & - & 1.95 & 117.00 & 117.00 & 61.50 & 55.80 & 1.90 \\
\hline $\mathrm{N}_{1} \mathrm{P}_{1}$ & 1.06 & 8.48 & 2.00 & 120.00 & 128.48 & 65.91 & 62.57 & 1.95 \\
\hline $\mathrm{N}_{1} \mathrm{P}_{2}$ & 2.05 & 16.40 & 2.03 & 121.80 & 138.20 & 65.97 & 73.23 & 2.09 \\
\hline $\mathrm{N}_{1} \mathrm{P}_{3}$ & 2.57 & 20.56 & 2.07 & 124.20 & 144.76 & 65.91 & 78.85 & 2.20 \\
\hline $\mathrm{N}_{1} \mathrm{P}_{4}$ & 4.24 & 33.92 & 1.67 & 100.20 & 134.12 & 65.98 & 68.14 & 2.03 \\
\hline $\mathrm{N}_{1} \mathrm{P}_{5}$ & 5.04 & 40.32 & 1.46 & 84.00 & 124.12 & 65.98 & 58.34 & 1.88 \\
\hline $\mathrm{N}_{2} \mathrm{P}_{0}$ & - & - & 2.69 & 161.40 & 161.40 & 62.80 & 98.60 & 2.57 \\
\hline $\mathrm{N}_{2} \mathrm{P}_{1}$ & 1.05 & 8.40 & 2.73 & 163.80 & 172.20 & 67.21 & 104.99 & 2.56 \\
\hline $\mathrm{N}_{2} \mathrm{P}_{2}$ & 2.21 & 17.68 & 2.75 & 166.20 & 183.88 & 67.07 & 116.81 & 2.74 \\
\hline $\mathrm{N}_{2} \mathrm{P}_{3}$ & 3.27 & 23.16 & 2.79 & 167.40 & 193.56 & 67.21 & 126.35 & 2.88 \\
\hline $\mathrm{N}_{2} \mathrm{P}_{4}$ & 4.39 & 35.12 & 2.40 & 144.00 & 179.12 & 67.28 & 111.84 & 2.66 \\
\hline $\mathrm{N}_{2} \mathrm{P}_{5}$ & 5.60 & 44.80 & 1.99 & 119.40 & 164.20 & 67.28 & 96.92 & 2.44 \\
\hline $\mathrm{N}_{3} \mathrm{P}_{0}$ & - & - & 2.67 & 160.20 & 160.20 & 63.23 & 96.97 & 2.53 \\
\hline $\mathrm{N}_{3} \mathrm{P}_{1}$ & 1.14 & 9.12 & 2.71 & 162.60 & 171.72 & 67.64 & 104.08 & 2.54 \\
\hline $\mathrm{N}_{3} \mathrm{P}_{2}$ & 2.14 & 17.12 & 2.86 & 171.60 & 188.72 & 67.50 & 121.22 & 2.79 \\
\hline $\mathrm{N}_{3} \mathrm{P}_{3}$ & 3.14 & 25.12 & 2.78 & 166.80 & 191.92 & 67.64 & 124.28 & 2.84 \\
\hline $\mathrm{N}_{3} \mathrm{P}_{4}$ & 4.37 & 34.96 & 2.35 & 141.00 & 175.96 & 67.61 & 108.35 & 2.60 \\
\hline $\mathrm{N}_{3} \mathrm{P}_{5}$ & 5.15 & 41.20 & 1.99 & 119.40 & 160.60 & 67.61 & 92.99 & 2.37 \\
\hline
\end{tabular}

Green fruit: Tk. $8.00 / \mathrm{kg}$, Seed: 60.00/kg ; Urea: Tk. $13.33 \mathrm{~kg}-\mathrm{N}$

* Detailed of treatments is given in Table 1.

Based on the above results, it might be suggested that picking of first 6 green fruits coupled with either 125 or $175 \mathrm{~kg} \mathrm{~N} / \mathrm{ha}$ gave the highest profit for okra production. But picking of 2-4 green fruits accompanied by $175 \mathrm{~kg} \mathrm{~N} / \mathrm{ha}$ was most suitable for quality seed production.

\section{LiterATURE CITED}

Ahmad, K. U. 1995. "Phal-Phul O Shak-Shabji (In Bengali)". 5th ed. Mrs Mumtaj Kamal, Mirpur, Dhaka, Bangladesh. 400 p.

Amjad, A., Anjum, M. A. and Hussain, S. 2001. Effect of different sowing dates and various doses of fertilizers on juvenility and productivity of okra. Pakistan J. Agril. Sci., 38(1-2), 29-32.

Bhuibhar, B. R., Mahakal, K. G., Kale, P. B. and Wankhade, S. G. 1989. Effect of time of sowing and number of pickings of green fruits on growth and seed yield of okra (Abelmoschus esculentus (L.)Moench). PKV Res. J., 13(1), 39-43.

Birbal, Nehra, B. K. and Malik, Y. S. 1995. Effect of spacing and nitrogen on fruit yield of okra (Abelmoschus esculentus (L.)Moench) cv. Varasha Uphar. Haryana Agric. Univ. J. Res., 25, 47-51.

Chattopadhyay, A. and Sahana, B. C. 2000. Response of okra seed crop to nitrogen and phosphorus fertilization in acidic soil of Old Alluvial Zone, West Bengal. Res. on Crops, 1(2), 176-180. 
Issac, S. R., Pushpakumari, R. and Saraswathy, P. 1998. Influence of green fruit picking and nutrient sources on fruit production in okra (Abelmoschus esculentus (L.)Moench). $\mathrm{J}$. Tropical Agric., 36(1-2), 50-52.

Khan, A. R. and Jaisal, R. C. 1988. Effect of nitrogen, spacing and green fruit pickings on the seed production of okra (Abelmoschus esculentus (L.)Moench). Veg. Sci., 15(1), 8-14.

Mozumder, S. N., Rahman, S. M. M., Rahman, M. H., Zaman, M. M. and Begum, K. 2002. Effect of N, P, K, and Mo on seed production of okra. Bangladesh J. Seed Sci. and Tech., 6(1 \& 2), 119-124.

Naik, L. B. and Srinivas, K. 1992. Influence of nitrogen and phosphorous on seed crop of okra. Indian J. Agron., 37(4), 769-771.

Nataraj, S.E., Kulkarni, G. N. Vyakaranahai, B. S. and Shasidhara, S. D. 1992. Effect of nitrogen levels and picking fresh fruits on seed quality of okra (Abelmoschus esculentus (L.)Moench). Karnataka J. Agril. Sci., 5(1), 51-53.

Paliwal, R., I. S. Naruka and J. K. Yadav. 1999. Effect of nitrogen and Sulphur on growth and yield of okra (Abelmoschus esculentus L. Moench.) cv. pusa sawani. Prog. Hort., 31(1-2), 9497.

Rahman, M. M., Roy, S. K. and. Quasem, A. 1992. Effect of plant population and nitrogen on the yield and yield attributes of okra. Bangladesh Agro. J., 4(1 \& 2), 7-12.

Rashid, M. M. 1999. "Shabjibiggayan (In Bengali)". Rashid publishing House, 94, Old DOHS, Dhaka-1206. p. 49.

Singh, R. K., Singh, G. P. and Singh, V. K. 1999. Effect of plant growth regulators and green fruit pickings on the seed production of Bhindi (Abelmoschus esculentus (L.)Moench). J. Applied Biol., 9(1), 31-34.

Talukder, A. S. M. H. M., Nabi, S. M., Ahmed, A. U., Anwar, M. N. and. Shaheed, M. M. A. 1999. Influence of N, P, K, Zn and Mo on okra in Grey Terrace Soil. Bangladesh J. Agril. Res., 24(3), 505-510.

Thakur, M. R. and S. K. Arora. 1986. Bhendi. In "Vegetable Crops in India" (T. K. Bose and M. G. Som, Eds.). Naya Prakash, 200, Bidhan Sarani, Calcata. pp. 613-622.

Uddin, M. M., Mondal, M. F., Samsuzzaman, S. and Siddique, M. A. 2006. Effect of different levels of nitrogen, phosphorous and potassium on the yield and quality of okra seed. In "Research Report on Horticultural Crops 2005-2006" (M. N. uddin, M. A. Rashid and M. S. Mullah (Eds.). Horticulture Research centre, Bangladesh Agricultural Research Institute, Joydebpur, Gazipur. pp. 108-114.

Velumani, N. P. and Ramaswamy, K. R. 1980. Effect of picking fruits on plant height and seed yeld in okra (Abelmoschus esculentus (L.)Moench). Seed Res., 8(1), 52-58.

Verma, I. M. and B. R. Batra. 2001. Effect of irrigation and nitrogen on growth and yield of okra. South Indian Hort., 49, 386-388. 